\title{
Community-acquired respiratory viruses after lung transplantation: common, sometimes silent, potentially lethal
}

\author{
Allan R Glanville
}

Community-acquired respiratory viruses (CARV) represent an ever present risk to the lung transplant (LTX) recipient. ${ }^{1}$ Alone among solid organ transplants, the lung allograft is exposed to the ambient environment with every breath; hence, it is exposed to CARV, which may trigger rejection. $^{2}$ Or at least we once thought despite some recent evidence to the contrary. ${ }^{3-5}$ The current article in the journal expands the previous work of the same group and provides a comprehensive analysis of 112 patients examined on 903 occasions over a 3-year period with a further 2-year follow-up to determine the presence and effects of CARV. ${ }^{6-8}$ The results are compelling in the main. CARV account for about $30 \%$ of all acute respiratory presentations after LTX and are a dominant cause of new respiratory symptoms with a risk of hospitalisation of $17-50 \%$ depending on type. Surprisingly, $10 \%$ of asymptomatic LTX recipients had a positive test for CARV at screening visits (predominantly rhinovirus), but whether their asymptomatic status represents the effects of maintenance immune suppressive therapy on the inflammatory response cannot be ascertained from the study.

The major strength of this prospective study is the exhaustive assessment of both symptomatic and asymptomatic LTX recipients throughout all seasons with prolonged follow-up. It would be hard to imagine a more detailed collation of symptom scores and surveillance for CARV. Limitations include the presence of missing data points in certain fields. For example, a number of patients (69/174) were not able to (or did not, for whatever reason) perform lung function at the time of CARV, which likely biases the observed fall in $\mathrm{FEV}_{1}$ towards an underestimate. Similarly, close scrutiny of appendix table 2 shows that when the Picornavirus group was excluded, the sample set of transbronchial lung biopsies (TBBx) was limited in patients with respiratory syncytial virus

Correspondence to Professor Allan R Glanville, The Lung Transplant Unit, Xavier 4, St. Vincent's Hospital, Victoria Street, Darlinghurst, NSW 2010, Australia; aglanville@stvincents.com.au
(2/11 cases), parainfluenza virus $(2 / 6$ cases), human metapneumovirus $(2 / 8$ cases), influenza (3/18), coronavirus $(3 / 22)$ and adenovirus/boca virus (0/4), making a sample size of $12 \mathrm{TBBx} / 69$ cases of CARV. Put simply, if you do not take a temperature, you cannot find a fever, and it is this observation that questions the biological plausibility of a statistical lack of association between CARV and acute rejection (AR). We need to know why the other 57 cases of CARV were not biopsied to ascertain the real potential for bias. Perhaps they were 'too sick' or perhaps they were perceived to be 'too well'. Not mandating a protocol TBBx undermines the potential ability to find an association if one exists. Until such time as we have a study with a high rate of contemporaneous TBBx with serial follow-up biopsies, the true association of CARV and AR must remain unclear. The more important question is, however, does it matter? Some evidence suggests even high-grade AR, if treated, may not be important in driving chronic lung allograft dysfunction (CLAD) manifest as the bronchiolitis obliterans syndrome (BOS). ${ }^{9}$ Untreated and persistent low-grade rejection may be associated with BOS. ${ }^{10}$ Lymphocytic bronchiolitis (LB) is associated with BOS, which is why the available data in this study are worthy of posthoc analysis. ${ }^{9}$ Excluding the Picornavirus group, the incidence of LB (B1R or higher grade) on TBBX in patients with CARV was $8 / 12$. B1R is known to be the harbinger of BOS. ${ }^{9}$ The purist might advise to exclude these biopsies from a BOS risk assessment due to 'concurrent infection', but if the pathology is identical it raises the issues of whether the long-term outcome would be different and also how often is the diagnosis of LB confounded by unsuspected CARV, especially if symptoms are nonspecific or attenuated by maintenance immune suppression. As the authors amply demonstrate, we now have the tools to allow a precise diagnosis of most CARV and it is tempting to recommend broad application of such an approach in all cases where bronchoscopy is performed after LTX. You cannot find what you do not look for.
There is building evidence to support the pathogenesis of obliterative bronchiolitis, the pathological hallmark of BOS, as the cumulative effect of an aberrant repair of an acute injury with downstream sequelae of epithelial damage. CARV cause an acute bronchitis/bronchiolitis, the severity of which depends on viral type and load. ${ }^{11}$ Subsequently, upregulation of antigens on epithelial surfaces leads to expression of both HLA class- 1 and class- 2 antigens plus or minus other cryptic antigens such as collagen-V, $\mathrm{k} \quad \alpha-1$ tubulin and bronchial microvascular endothelial antigens. ${ }^{12}$ This prepares the way for a subsequent alloimmune and autoimmune response targeted to the airway epithelium and/or microvasculature leading to chronic lung allograft rejection. ${ }^{13}$ The current study rightly does not address the propensity for the late development of CLAD after CARV, which has been shown well in prior studies. 12410 14-16

It does show that rhinovirus should not be sneezed at! In fact, the preponderance of Picornavirus infections (60\% of CARV in this series) allows greater insight into the potential deleterious effects of these ubiquitous pathogens. Once thought to have a lower tropism for the lower respiratory tract, it is apparent that LTX recipients are at increased risk with $18 \%$ admitted to hospital. Persistent rhinovirus infection in particular may be a surrogate biological marker of over immunosuppression as suggested by the higher calcineurin inhibitor levels in the CARV group. Not all CARV are the same as nicely described in table 3, and further subdivision of the Picornavirus group in particular may bring additional insights regarding severity and persistence of symptoms noting a recent report of more severe symptoms in human rhinovirus type $\mathrm{C} .{ }^{17}$ Finally, the authors avoid discussing the thorny clinical dilemma of whom to treat and how to treat. That is a story for another day, but there are potentially exciting times ahead. ${ }^{18}$

Competing interests None.

Provenance and peer review Commissioned; externally peer reviewed.

To cite Glanville AR. Thorax 2014;69:1-2.

Published Online First 11 November 2013

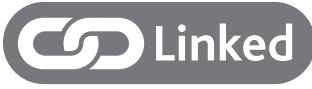

- http://dx.doi.org/10.1136/thoraxjnl-2013-203581

Thorax 2014;69:1-2.

doi:10.1136/thoraxjnl-2013-204327 


\section{REFERENCES}

1 Gottlieb J, Schulz TF, Welte T, et al. Communityacquired respiratory viral infections in lung transplant recipients: a single season cohort study.

Transplantation 2009;87:1530-7.

2 Billings JL, Hertz MI, Savik K, et al. Respiratory viruses and chronic rejection in lung transplant recipients. J Heart Lung Transplant 2002;21: 559-66.

3 Vilchez R, McCurry K, Dauber J, et al. Influenza and parainfluenza respiratory viral infection requiring admission in adult lung transplant recipients. Transplantation 2002;73:1075-8.

4 Kumar D, Husain S, Chen MH, et al. A prospective molecular surveillance study evaluating the clinical impact of community-acquired respiratory viruses in lung transplant recipients. Transplantation 2010;89: 1028-33.

5 Sayah DM, Koff JL, Leard LE, et al. Rhinovirus and other respiratory viruses exert different effects on lung allograft function that are not mediated through acute rejection. Clin Transplant 2013;27:E64-71. doi: 10.1111/ctr.12054

6 Bridevaux PO, Aubert JD, Soccal PM, et al. Incidence and outcomes of respiratory viral infections in lung transplant recipients: a prospective study. Thorax 2014;69:32-8
7 Soccal PM, Aubert JD, Bridevaux PO, et al. Upper and lower respiratory tract viral infections and acute graft rejection in lung transplant recipients. Clin Infect Dis 2010;51:163-70.

$8 \mathrm{Vu} \mathrm{DL}$, Bridevaux PO, Aubert JD, et al. Respiratory viruses in lung transplant recipients: a critical review and pooled analysis of clinical studies. Am J Transplant 2011;11:1071-8.

9 Glanville AR, Aboyoun CL, Havryk A, et al. Severity of lymphocytic bronchiolitis predicts long-term outcome after lung transplantation. Am J Respir Crit Care Med 2008;177:1033-40.

10 Hopkins PM, Aboyoun CL, Chhajed PN, et al. Association of minimal rejection in lung transplant recipients with obliterative bronchiolitis. Am J Respir Crit Care Med 2004;170:1022-6.

11 DeVincenzo JP, Wilkinson T, Vaishnaw A, et al. Viral load drives disease in humans experimentally infected with respiratory syncytial virus. Am J Respir Crit Care Med 2010;182:1305-14.

12 Tiriveedhi V, Angaswamy N, Brand D, et al. A shift in the collagen $V$ antigenic epitope leads to $T$ helper phenotype switch and immune response to self-antigen leading to chronic lung allograft rejection. Clin Exp Immunol 2012;167:158-68.

13 Hachem RR, Tiriveedhi V, Patterson GA, et al. Antibodies to K-alpha 1 tubulin and collagen $V$ are associated with chronic rejection after lung transplantation. Am J Transplant 2012;12: 2164-71.

14 Uckay I, Gasche-Soccal PM, Kaiser L, et al. Low incidence of severe respiratory syncytial virus infections in lung transplant recipients despite the absence of specific therapy. J Heart Lung Transplant 2010;29:299-305.

$15 \mathrm{Ng} \mathrm{BJ}$, Glanville AR, Snell G, et al. The impact of pandemic influenza A H1N1 2009 on Australian lung transplant recipients. Am J Transplant 2011;11: 568-74.

16 Khalifah AP, Hachem RR, Chakinala MM, et al. Respiratory viral infections are a distinct risk for bronchiolitis obliterans syndrome and death. Am J Respir Crit Care Med 2004;170:181-7.

17 Cox DW, Bizzintino J, Ferrari G, et al. HRV-C infection in young children with acute wheeze is associated with increased acute respiratory hospital admissions. Am J Respir Crit Care Med Published Online First: 30 Aug 2013. doi:10.1164/ rccm.201303-04980C

18 Zamora MR, Budev M, Rolfe $M$, et al. RNA interference therapy in lung transplant patients infected with respiratory syncytial virus. Am J Respir Crit Care Med 2011;183: 531-8. 